\title{
Acidos grasos omega-3 en la nutrición ¿como aportarlos?
}

\author{
Omega-3 fatty acids in nutrition, \\ how to get them?
}

\begin{abstract}
The benefits derived from the consumption of omega-3 fatty acids EPA and DHA are strongly documented existing recommendations for their intake. Marine products, specifically fish, are the most obvious recommendation. However, the availability of this resource is at present increasingly limited. Marine oils rich in omega-3 fatty acids are also increasingly scarce and very expensive. Fish oil, which is highly susceptible to oxidation, can be protected by procedures of encapsulation, microencapsulation o nanoencapsulation, but the amount of omega-3 fatty acids provided by the last two technologies is very small, and capsules are not well tolerated by everyone. Marine algae and krill oil are another two alternatives for omega-3 availability and consumption. However, algae culture is expensive and krill oil is scarce and of high cost. The possibility to obtain residual phospholipids from fish meal is a technological alternative now under experimental investigation. The harvesting of terrestrial vegetables which produces fruits and/ or seeds with a high content of alpha linolenic acid (ALA), the metabolic precursor of EPA and DHA, is a novel interesting alternative to provide omega-3 fatty acids. However, it is necessary to demonstrate how effective the metabolic bioconversion of ALA into EPA and DHA is before encouraging the harvest and production of these vegetables oils, this being a pending challenge. The present work critically reviews the alternatives at present available to incorporate omega-3 fatty acid in our diet.

Key words: omega-3 fatty acids, availability of omega-3 fatty acids, procedures to obtain omega-3 fatty acids, new sources of omega-3 fatty acids.
\end{abstract}

\section{INTRODUCCIÓN}

Los beneficios derivados del consumo habitual de los ácidos grasos omega-3 de origen marino están suficientemente documentados con sólida y abundante literatura científica que respalda su efectividad (1). Estos ácidos grasos son principalmente el ácido eicosapentaenoico (C20:5, EPA) y el ácido docosahexaenoico (C22:6, DHA). Originalmente, se atribuyó al EPA, a través de su transformación en diferentes eicosanoides (compuestos derivados de 20 átomos de carbono), los beneficios cardiovasculares (efectos hipotrigliceridémicos, hipocolesterolémicos, antitrombóticos, antiinflamatorios, antiarritmicos, entre otros) $(1,2)$ y al DHA una importante función en el desarrollo, función y protección del sistema nervioso $(3,4)$. A la luz del conocimiento actual, la interpretación de los efectos individuales de ambos ácidos grasos es mucho más compleja. La postura científica más reciente refiere los
Alfonso Valenzuela B. $(1,2)$

Rodrigo Valenzuela B. (3)

(1) Instituto de Nutrición y Tecnología de los Alimentos, Universidad de Chile, Santiago, Chile. (2) Facultad de Medicina, Universidad de los Andes, Santiago, Chile (3) Escuela de Nutrición y Dietética, Facultad de Medicina, Universidad de Chile, Santiago, Chile.

Dirigir la correspondenica a: Profesor Alfonso Valenzuela $B$ INTA - Universidad de Chile El Líbano 5524-Macul, Santiago, Chile Casilla 138-11

Fono: 56-2-29781449, Fax: 56-2-22214030 e-mail: avalenzu@inta.uchile.cl

Este trabajo fue recibido el 2 de Noviembre de 2013 y aceptado para ser publicado el 16 de Mayo de 2014.

efectos fisiológicos de los ácidos grasos omega-3 a derivados metabólicos del DHA (docosanoides o derivados de 22 átomos de carbono, resolvinas y neuroprotectinas) (5) posicionando al EPA solo como un intermediario en la formación del DHA. De hecho, nuestro organismo no acumula EPA, salvo que lo consumamos en cantidades relativamente importantes (6). Independientemente que sea el EPA o el DHA, o ambos ácidos grasos, o sus derivados metabólicos los que aportan los beneficios de salud atribuidos a estos ácidos grasos, la recomendación es consumirlos con frecuencia (entre 250 mg a 2 g/día) ya que sus efectos biológicos representan un beneficio para nuestra salud y nutrición (7), aunque ya surgen alarmas, como se discutirá más adelante (8). El tema entonces es ¿Cómo consumir ácidos grasos omega-3 de origen marino? En este trabajo, se discuten las diferentes alternativas que actualmente están disponibles o en desarrollo. 


\section{¡A COMER PESCADO!}

Sí, es la recomendación obvia de las autoridades de salud y nutrición, especialmente el consumo de pescados grasos o "azules", ya que constituyen la alternativa más sabrosa (si están bien preparados) y nutricionalmente más adecuada para obtener ácidos grasos omega-3 (7). De hecho, los evolucionistas plantean que nuestro cerebro se diferenció del cerebro del resto de los primates, incluso de nuestros parientes más cercanos, los chimpancés, debido que nuestros antepasados se acercaron al mar e incluyeron en la dieta pescados y otros productos del mar (9). La adquisición de mayores cantidades de ácidos grasos omega-3 probablemente comenzó con el desarrollo del carnivorismo, ya que el uso de herramientas tan simples como piedras o grandes huesos, permitió a nuestros ancestros el acceso al cerebro y al tuétano de los animales capturados por verdaderos carnívoros (hienas, buitres, entre otros) (10). No éramos cazadores, éramos carroñeros o más sutilmente, carnívoros mal adaptados.

Las estadísticas del bajo consumo de pescado en nuestro país y en otros países de la región ya son conocidas y no se espera que puedan mejorar (figura 1). ¿Por qué comemos tan poco pescado? Las razones son varias: no siempre es fácil de conseguir, se deteriora con rapidez, tiene bajo rendimiento como alimento ya que en su preparación no se aprovechan la cabeza, cola, vísceras, etc., además de la "baja saciedad generada" en comparación a otras carnes y también es más costoso en comparación con otras carnes (pollo, cerdo, pavo). Las numerosas campañas para incrementar el consumo de pescado no han resultado efectivas.

A este aspecto de índole nutricional se suma uno de tipo productivo-económico. La tecnificación de la pesca industrial ha sobrepasado la capacidad reproductiva y de renovación del recurso pesquero, con lo cual hoy se captura mucho menos peces y cada vez de menor tamaño. Un reciente informe de la Organización para los Alimentos y la Agricultura (FAO, 2010)
(11) plantea que en 25 años más un número importante de especies marinas, entre ellas de varios peces, estarán al borde de la extinción si no se modifican las políticas de pesquería de los grandes actores en este rubro (Chile, Perú, Noruega, Dinamarca, China, Japón, Estados Unidos, entre otros). Además, actualmente es más rentable la captura de peces para la obtención de harina y aceite de pescado que su derivación para consumo directo o conservas (12). La industria acuicultora ha generado una gran demanda por estos dos insumos, harina y aceite de pescado. FAO (11) anticipa que el futuro en la disponibilidad de peces para el consumo directo derivará de la acuicultura, pero esta situación crea una paradoja. El enorme crecimiento de la acuicultura ha generado una gran demanda de harina y aceite de pescado, demanda a la que la industria pesquera no puede responder, generando un alza de los precios de ambos insumos y una escases cada vez más preocupante (12).

La industria acuicultora ha buscado remplazar los ácidos grasos omega-3 marinos por ácidos grasos omega-3 vegetales (alfa linolénico, C18:3, ALA), principalmente a base de aceite de soja o canola, que no es lo mismo ya que el salmón, que es el principal producto de la acuicultura, requiere el EPA y DHA preformado ya que no puede transformar el ALA en estos ácidos grasos (13). ¿Qué ha ocurrido?, nuestros salmones hoy día aportan poco EPA y DHA y sí contienen ALA, o sea se están transformando en "vegetarianos". El salmón ya no es el "banquete de salud" como lo definimos años atrás (14). Algunos mariscos, como el ostión y el mejillón, son también buenas fuentes de EPA y DHA, aunque no comparables al aporte de peces como el atún, la anchoa, la sardina o el modesto jurel, quizás la mejor fuente de ácidos grasos omega-3 actualmente disponible (15).

En conclusión, el aporte de ácidos grasos omega-3 a través de los productos del mar, al parecer no constituirá en el futuro la mejor y más abundante fuente de estos ácidos grasos.

\section{Consumo de pescado en algunos países}

\begin{tabular}{|l|c|}
\hline \multicolumn{1}{|c|}{ País } & Consumo (Kg/ cápita/ año) \\
\hline Islandia & 90 \\
Portugal & 76 \\
Japón & 64 \\
Noruega & 53 \\
España & 44 \\
Venezuela & 12,5 \\
Uruguay & 10,4 \\
Argentina & 9,5 \\
Brasil & 8,3 \\
México & 8,0 \\
Perú & 7,0 \\
Chile & 5,8 \\
Colombia & 4,8 \\
\hline
\end{tabular}




\section{¿Y EL ACEITE DE PESCADO?}

Nuestras abuelitas sabían que el aceite de hígado de bacalao tenía muchas virtudes saludables, por lo cual era tradicional que se lo dieran diariamente a nuestros padres o a alguno de nosotros. La recomendación era por su alto contenido de vitamina $A$ y $D$, ya que nada se sabía sobre las virtudes de los ácidos grasos omega-3 contenidos en el aceite, el cual no era muy agradable. La tecnología actual permite refinar y desodorizar los aceites marinos transformándolos en productos consumibles, aunque con ciertas limitaciones, ya que con el tiempo estos aceites revierten su característico olor y tienden a deteriorarse por oxidación (12).

Uno de los grandes problemas tecnológicos y por derivación, alimentarios, es la alta susceptibilidad de los aceites marinos a desarrollar deterioro oxidativo y organoléptico (16). La industria farmacéutica y nutracéutica ha buscado alternativas. Se pueden preparar aceites saborizados y edulcorados que son medianamente aceptados por los consumidores, particularmente por los niños, a quienes estos productos van dirigidos principalmente. Una alternativa más atractiva desde el punto de vista del consumo, es preparar emulsiones de aceites marinos en agua, jugos o lácteos. Utilizando emulsionantes adecuados es posible incorporar en forma estable aceites marinos a estos alimentos, aunque las cantidades de ácidos grasos omega-3 que se logran aportar son sustancialmente menores que las que aporta el aceite como tal. Productos de este tipo ya se comercializan en diferentes países (Francia, Alemania, Estados Unidos, Argentina, Brasil, entre otros). Esta modalidad solo permite la adición de aceite a productos en base líquida. La recomendación de consumir altas cantidades de ácidos grasos omega-3 (sobre 2 o 3 gramos/día) para conseguir una efectiva protección cardiovascular y del sistema nervioso (17), actualmente despierta preocupación en algunos medios científicos a raíz de la información que niveles plasmáticos altos de DHA, que obviamente derivan de un alto consumo de este ácido graso, pueden facilitar el desarrollo de cáncer prostático agresivo $(18,19)$. Será necesario contar con más información científica y epidemiológica para resolver sobre esta preocupante observación.

La alternativa más clásica para consumir aceites marinos son las conocidas cápsulas blandas de aceite de pescado. Estos productos, generalmente contienen entre $500 \mathrm{mg}$ a $1000 \mathrm{mg}$ de aceite de pescado que en promedio aportan 250 a 300 mg de EPA + DHA en proporciones que varían de acuerdo al origen del aceite (20). Algunas de estas cápsulas se saborizan para hacer más aceptable su consumo, ya que es habitual que produzcan cierta flatulencia o "repitan". Esta modalidad de consumo está dirigida a los adultos, ya que los niños difícilmente aceptarán o podrán consumir estas cápsulas que son de tamaño relativamente grande. En algunos países (Alemania, Dinamarca) se han desarrollado "gomitas" con omega-3, las que son bastante agradables de consumir tanto por adultos como para niños.

Una alternativa de reciente desarrollo es la microencapsulación de aceite de pescado (21). Mediante un tratamiento tecnológico que requiere una microdispersión del aceite y su atrapamiento en polímeros de maltodextrina u otros derivados del almidón o celulósicos, es posible preparar micro partículas de 1-5 $\mu \mathrm{m}$ que en peso contienen hasta un $20 \%$ de aceite. Se trata, entonces, de un producto en polvo que puede ser adicionado a alimentos en base seca, como es el caso de leche en polvo, cereales, alimentos infantiles, fórmulas lácteas, entre otras. Son productos de relativo alto costo, pero se estima que en la medida que se masifique su aplicación se lograrán costos que puedan ampliar sus aplicaciones, porque se trata de una excelente alternativa que aporta estabilidad al aceite, incluso cuando el producto se reconstituye a una forma líquida (20).

Más recientemente, utilizando una tecnología de micro coacervación se han desarrollado nanopartículas conteniendo aceites marinos o concentrados de ácidos grasos omega-3 que permiten, incluso, su adición al agua (agua mineral, jugos traslúcidos) sin que se genere opacidad (22). La desventaja es que se puede obtener sólo una pequeña inclusión de ácidos grasos omega-3, no superior a 10-20 mg por porción de consumo.

\section{ÁCIDOS GRASOS OMEGA-3 DERIVADOS DE ACEITES MARINOS}

El requerimiento de mayores concentraciones de ácidos grasos omega-3, actualmente recomendadas para la prevención de enfermedades cardiovasculares y particularmente neurodegenerativas y del comportamiento (17), ha motivado el desarrollo de procesos que permiten obtener altas concentraciones de ácidos grasos omega-3 ya sea de EPA, DHA o de ambos.

El proceso consiste en separar los ácidos grasos de las estructura triglicerídica de los aceites marinos, transformándolos en esteres del etanol (etil-esteres). Este proceso se puede realizar en forma química o idealmente mediante enzimas que liberan específicamente los ácidos grasos omega-3 por su estéreo especificidad desde los triglicéridos que componen el aceite (23). Los etil-esteres se separan mediante un proceso de destilación molecular obteniéndose concentraciones hasta de $90 \%$ de cada ácido graso (EPA o DHA). Estos etil-esteres se pueden adicionar a una gran variedad de productos, ya sea en base oleosa o en base acuosa, en este caso utilizando emulsionantes adecuados. También se pueden encapsular, microencapsular o nanoencapsular. Sin embargo, existe cuestionamiento sobre la real biodisponibilidad de los ácidos grasos omega-3 en la forma de etil-esteres, ya que estos productos son mal sustrato para las lipasas intestinales, lo que reduciría notablemente su absorción en el tubo digestivo (24). Como alternativa, los etil-esteres se pueden volver a transformar nuevamente en triglicéridos, obteniéndose así aceites con alto contenido de EPA, de DHA o de ambos ácidos grasos (25). Actualmente se ofrecen comercialmente aceites con un $70 \%$ de algunos de estos ácidos grasos o combinaciones de ambos.

\section{ÁCIDOS GRASOS OMEGA-3 NO DERIVADOS DE PECES: LAS MICRO ALGAS}

Los ácidos grasos omega-3 no son sintetizados por los animales marinos, ellos los incorporan como parte de la cadena trófica alimentaria desde los vegetales marinos quienes tienen la maquinaria metabólica para realizar la síntesis de novo de estos ácidos grasos (26). Dentro de la abundancia biológica del micro plancton, las micro algas son particularmente efectivas en el proceso de biosíntesis de ácidos grasos omega-3. Especies como Cryptheconium, Mortierella y Schizochytrium se cultivan artificialmente en grandes bioreactores y de las cuales se extrae aceite que es particularmente rico en fosfolípidos que contienen, ya sea DHA (estas algas no producen EPA o lo hacen en muy baja concentración) o ácido araquidónico (C20:4, AA), un ácido graso omega- 6 que también tiene importancia nutricional. Los fosfolípidos que contienen tanto DHA como AA, o ambos ácidos grasos, se utilizan en la preparación de fórmulas lácteas que sustituyen a la leche materna (que naturalmente contiene ambos ácidos grasos (27), se pueden encapsular, micro encapsular y es posible que en el futuro se puedan nano encapsular. 
Las ventajas del uso de las micro algas para la obtención de ácidos grasos omega-3 son varias: se trata de un recurso renovable ya que provienen de cultivos artificiales; el aceite que se obtiene es rico en fosfolípidos, cualidad que se discutirá en las siguientes secciones; dicho aceite no tiene "olor a pescado", con lo cual es organolépticamente más aceptable que el aceite de pescado; la estabilidad de los ácidos grasos poliinsaturados incorporados a los fosfolípidos es mayor que cuando estos ácidos grasos están incorporados a los triglicéridos, lo cual aporta una ventaja adicional a la estabilidad oxidativa de los productos que contienen este tipo de aceites. Otra ventaja de los fosfolípidos es que estos, a diferencia de los triglicéridos, son polares con lo cual son emulsionables e incluso actúan como emulsionantes, propiedad que permite su incorporación a matrices alimentarias acuosas, siendo su adición mucho menos compleja que la de los aceites en base a triglicéridos (28). Desde el punto de vista nutricional, la ventaja más destacable es que los ácidos grasos omega-3 son mucho más biodisponibles cuando se aportan en la forma de fosfolípidos que en la forma de triglicéridos (28). Este último aspecto es muy relevante y ha reorientado la investigación y el desarrollo de productos conteniendo ácidos grasos omega-3. Actualmente el interés se centra en la obtención de fosfolípidos marinos, más que en los aceites marinos en base a triglicéridos.

Derivado del interés en los fosfolípidos, un constituyente del zoo plancton, el krill (Euphausia superba) también resulta muy interesante como fuente de ácidos grasos omega-3 (29). Este micro crustáceo cuando es sometido a presión (prensado) produce un aceite de intenso color rojo que contiene hasta un $60 \%$ de fosfolípidos. El color rojo lo produce un carotenoide, la astaxantina, que actúa como antioxidante del producto y que además es muy demandado para aportar color a los salmones de cultivo y más recientemente en medicina humana por sus potentes efectos antinflamatorios y antioxidantes (30). La captura de krill es estacional y sujeta a controles internacionales debido a que es el principal alimento de cetáceos y otros animales marinos (focas, pingüinos, aves), con lo cual no es un recurso de alta disponibilidad y en un futuro próximo su captura será muy controlada e incluso prohibida. Los fosfolípidos derivados del krill tienen un alto valor de mercado, sobre US\$ $75 / \mathrm{kg}$ y varias empresas, principalmente europeas, los ofrecen a la industria farmacéutica y nutracéutica (31).

\section{NUEVAS ALTERNATIVAS PARA LA OBTENCIÓN DE FOSFOLÍPIDOS MARINOS}

En razón del interés actual por los fosfolípidos, a nivel aún experimental, se están explorando otras alternativas para su obtención. Por ejemplo, a partir de los subproductos de la industria acuicultora (vísceras, cabezas, colas, esquelones), principalmente del salmón, que se utilizan para la obtención de harina y aceite, es posible extraer fosfolípidos con interesante contenido de ácidos grasos omega-3.

Para esto, estos subproductos se someten a hidrólisis controlada con enzimas proteolíticas que liberan los fosfolípidos desde las estructuras proteicas de las membranas de las cuales forman su estructura matricial. Estos fosfolípidos se precipitan selectivamente y se concentran hasta porcentajes variables según el requerimiento de concentración que se desee (22).

Se comentó que los salmones actualmente aportan un bajo contenido de ácidos grasos omega-3, esto debido a la modificación de la dieta con la incorporación de aceites vegetales terrestres que aportan ALA y no EPA y DHA. Sin embargo, esto no ocurre con los fosfolípidos, ya que el pez concentra su capacidad de retención de estos ácidos grasos casi selectivamente en los fosfolípidos que son parte de su estructura celular y no en los triglicéridos que constituyen su reserva energética. La figura 2 muestra las alternativas alimentarias y tecnológicas actualmente disponibles para el aporte nutricional de ácidos grasos omega-3 EPA y DHA.

\section{Diferentes formas de aportar ácidos grasos omega -3 a partir de productos marinos}

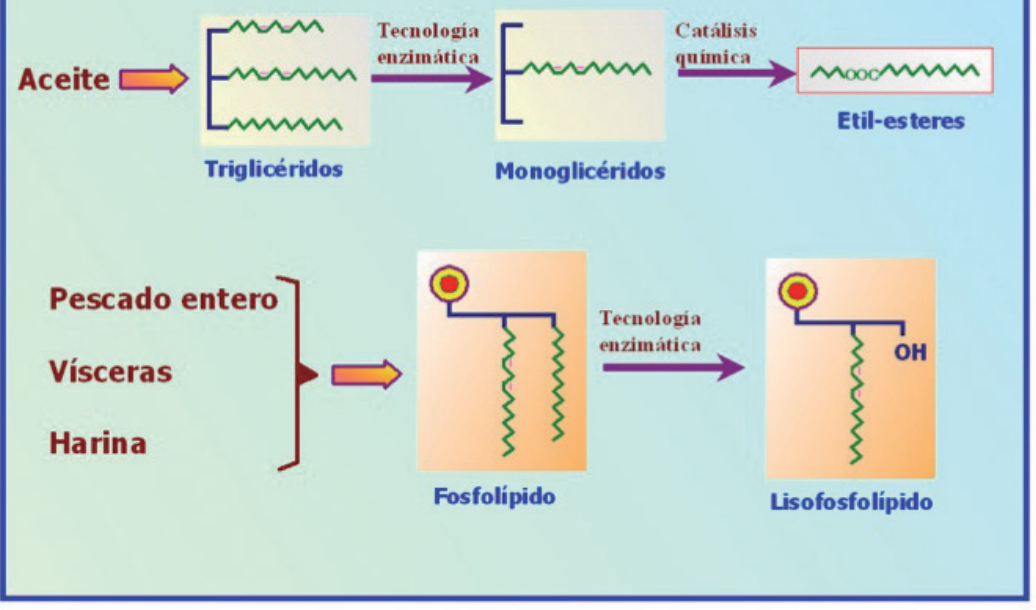




\section{ACEITES DE ORIGEN TERRESTRE QUE APORTAN ÁCIDOS GRASOS OMEGA-3}

Los aceites derivados de semillas vegetales de origen terrestre no contienen EPA ni DHA, pero algunos de estos aceites tienen un alto contenido de ALA, el precursor natural del EPA y DHA. Este es el caso del aceite de lino (o linaza), muy utilizado industrialmente en la fabricación de barnices y pinturas por su efecto secante (derivado de su alto contenido de ALA, 60\%) pero que se utiliza muy poco como alimento por su alta inestabilidad. Actualmente se visualiza a este aceite, debidamente procesado y estabilizado con antioxidantes, como una alternativa viable para aportar nutricionalmente ácidos grasos omega-3. Sin embargo, queda aún por demostrar qué cantidad de ALA es necesario consumir para lograr una adecuada conversión del ácido graso en EPA o más importantemente, en DHA.

Experimentalmente, se ha demostrado que la bioconversión del ALA en EPA o DHA es baja (menos del 5\%) (32), pero la disponibilidad de aceites que permiten consumir cantidades mayores de ALA en alimentos o como suplementos, puede compensar el requerimiento. Los autores de este artículo estamos trabajando en demostrar la efectividad de esta biotransformación tanto en el modelo animal como humano.

Además del conocido aceite de lino, en Sudamérica ancestralmente se han producido y consumido semillas que contienen un alto aporte de ALA, es el caso de los aceites de sacha inchi, chía y rosa mosqueta, entre otros. El aceite de sacha inchi, se obtiene de los frutos de la planta (Plukenetia volubilis) originaria del Perú y aporta hasta un $46 \%$ de ALA. EI aceite de chía se obtiene de la planta del mismo nombre (Salvia hispánica), originaria de México, Guatemala y Honduras. Este aceite aporta un $63 \%$ de ALA.

Recientemente se inició en Chile el cultivo experimental de chía. El aceite de rosa mosqueta, que se obtiene de las semillas de la misma planta (Rosa cannina) es producido en
Chile con fines casi exclusivamente cosméticos. Sin embargo, su contenido de ALA (30\%) también lo hace una interesante fuente nutricional de este ácido graso. Estos aceites, juntos con otros de origen europeo y/o asiático (camelina, perilla, entre otros), constituyen lo que hoy día se llaman "aceites de nuevo desarrollo" (33), no porque sean de reciente descubrimiento, ya que se utilizan desde cientos de años por las poblaciones originarias, sino que actualmente constituyen una interesante alternativa para aportar ALA como precursor de los ácidos grasos oemga-3 EPA y DHA, cada vez de menor disponibilidad y mayor costo. La figura 3 muestra el contenido de ácidos grasos omega-3 (ALA) de estos aceites.

Sin embargo, está pendiente el desafío de demostrar cuan eficientes son estos aceites, cuando son aportados en cantidades adecuadas, para sustituir el consumo directo de aceites de origen marino y poder aportarnos los beneficios ya demostrados de la ingesta de EPA y/o DHA. Al respecto, nuestro grupo de investigación ha demostrado en forma preliminar el incremento en los niveles de EPA y DHA en diferentes tejidos de ratas alimentadas con aceite de chía (34), observándose, además, un aumento en la expresión hepática de genes regulados por estos ácidos grasos (35).

\section{CONCLUSIONES}

Es cada vez más complejo consumir ácidos grasos omega-3 de origen marino. La fuente natural, los animales marinos, particularmente los peces, es cada vez más escasa. La producción de aceites marinos ricos en EPA y DHA también es cada vez menor debido a la poca disponibilidad del recurso marino. Las microalgas, fuentes naturales de DHA y también de EPA son de alto costo de producción ya que requieren de complejas instalaciones industriales. Los fosfolípidos marinos han adquirido actualmente mucha importancia porque se considera que son productos de mucho mayor biodisponibilidad que los triglicéridos. Sin embargo, la única fuente importante

\section{FIGURA 3}

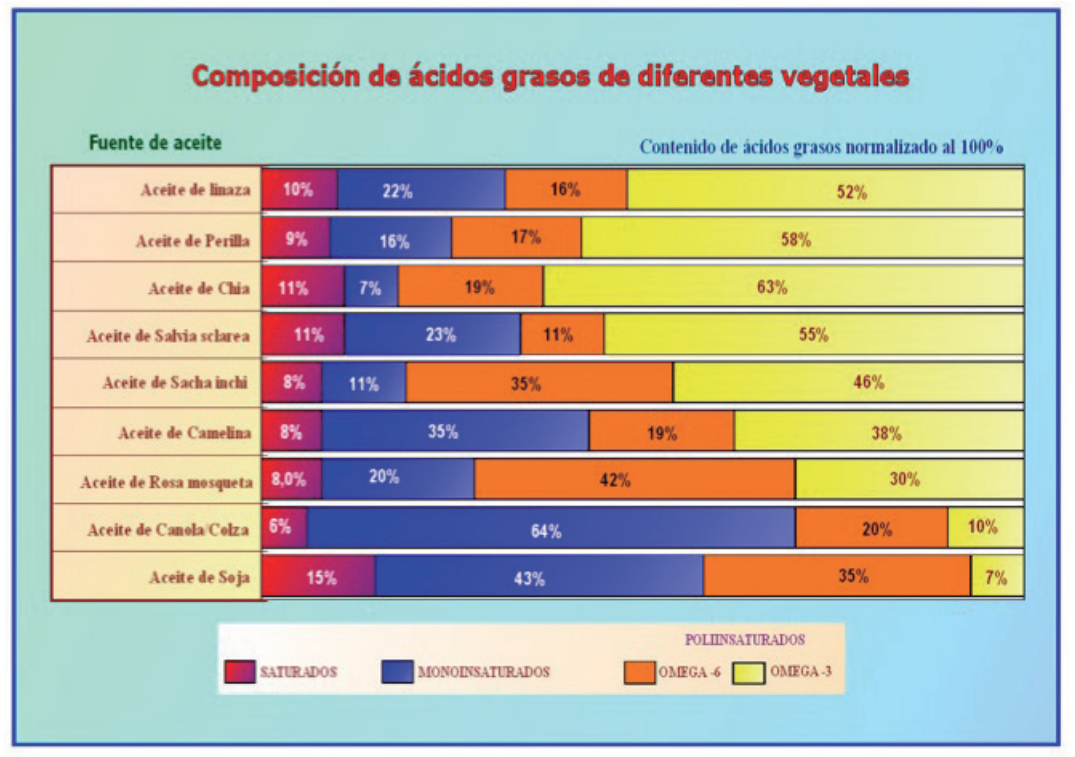


para obtenerlos son las micro algas, ya comentadas y el aceite de krill, el cual es escaso, de alto costo y probablemente sujeto a restricciones en un futuro próximo. La posibilidad de obtener fosfolípidos ricos en omega-3 a partir de deshechos de la industria acuicultora o de la harina de pescado, aún es experimental.

Actualmente el interés está en aceites de origen vegetal terrestre que aportan importantes cantidades de ALA, el precursor del EPA y del DHA. Sin embargo, queda aún por demostrar que el consumo de ALA, en cantidades relativamente altas, pueda ser convertido en nuestro organismo a los ácidos grasos omega-3 metabólicamente activos. Este es un desafío actualmente vigente y muchos grupos de investigación, incluido el nuestro, busca una respuesta a esta incógnita metabólica. Si se verifica la biotransformación del ALA a EPA y DHA, se abrirá una excelente posibilidad para estimular el cultivo de plantas productoras de frutos y/o semillas ricas en ALA, con lo cual estos productos, los frutos, las semillas o los aceites extraídos de ellas, pueden llegar a ser nutrientes que mejoren sustancialmente el desequilibrio omega-6/omega-3 que afecta al grueso de la población occidental. Sin embargo de ser válida esta hipótesis, además del compromiso académico e industrial para conocer y producir estos vegetales será también necesario disponer de un marco regulatorio que permita una adecuada y atractiva comercialización de alimentos con ácidos grasos omega-3, tema aún pendiente en Chile.

\section{RESUMEN}

Los beneficios derivados del consumo de ácidos grasos omega-3 EPA y DHA de origen marino están sólidamente documentados, por lo cual existen recomendaciones para su consumo. La recomendación es consumir productos del mar, específicamente pescado. Sin embargo la disponibilidad de este recurso es cada vez menor. Los aceites marinos, ricos en ácidos grasos omega-3 son también cada vez más escasos y de mayor costo. El aceite de pescado, altamente inestable a la oxidación, se puede encapsular, micro encapsular o nano encapsular, aunque los aportes de ácidos grasos de estas dos últimas tecnologías son pequeños y las cápsulas no son toleradas por todas las personas. Otras alternativas para la producción de ácidos grasos omega-3 son las algas marinas o el aceite de krill. El cultivo de algas es de alto costo y el aceite de krill es escaso y de alto valor. La posibilidad de obtener fosfolípidos residuales a partir de la harina de pescado es actualmente una alternativa que se estudia experimentalmente. El cultivo de vegetales terrestres que producen frutos y/o semillas y la extracción de aceite con alto contenido de ácido alfa-linolénico $(A L A)$, el precursor metabólico del EPA y del DHA, representa una alternativa interesante de estudiar. Sin embargo, es necesario conocer la efectividad de la biotransformación del ALA en EPA y DHA para propiciar el cultivo y producción de estos aceites vegetales. Este es un desafío pendiente. Este artículo realiza una revisión crítica de las alternativas actuales para obtener ácidos grasos omega-3 de origen marino para nuestra nutrición.

Palabras clave: ácidos grasos omega-3, disponibilidad de ácidos grasos omega-3, procedimientos para obtener ácidos grasos omega-3, nuevas fuentes de ácidos grasos omega-3.

\section{BIBLIOGRAFÍA}

1. Mozaffarian D, Wu J. (n-3) Fatty acids and cardiovascular health: Are effects of EPA and DHA shared or complementary? J Nutr. 2012; 142: 614S-25S.

2. Calder P. Omega-3 polyunsaturated fatty acids and in- flammatory processes: nutrition or pharmacology? Brit J ClinPharmacol. 2012; 75: 645-62.

3. Richardson A, Burton J, Thees S, et al. Docosahexaenoic acid for reading, cognition and behavior in children aged 7-9 years: A randomized, controlled trial (The DOLAB Study). Plos One 2012; 7: e43909, 1-14.

4. Cunnane S, Plourde M, Pifferi F, et al. Fish, docosahexaenoic acid and Alzheimer's disease. Prof Lipid Res. 2009; 48: 239-56.

5. Crawford M. Docosahexaenoic acid in neural signaling systems. Nutr Health. 2006; 18: 263-76.

6. Arterburn L, Hall E, Oken H. Distribution, interconversion, and dose response of $n-3$ fatty acids in humans. Am J Clin Nutr. 2006; 83: S1467-76.

7. Larsen $R$, Eilertsen $K$, Elvevoll E. Health benefits of marine foods and ingredients. Biotechnol. Adv. 2011; 29: 508-18.

8. Chua $M$, Sio $M$, Sorongon $M$, et al.The relevance of serum levels of long chain omega-3 polyunsaturated fatty acids and prostate cancer risk. A meta-analysis. Can Urol Assoc J 2013; 7: E333-E43.

9. Simopoulos A. Evolutionary aspects of diet, the olega-6/ omega-3 ratio and genetic variation: nutritional implications for chronic diseases. Biomed Pharmacol. 2006; 60: 502-07.

10. Valenzuela A. ¿Por qué comemos lo que comemos? Rev Chil Nutr. 2011; 38: 198-209.

11. Joint FAO/WHO Expert Consultation on the risks and benefits of fish consumption. FAO Fisheries and Aquaculture Report 978. Rome, 2010.

12. Valenzuela A, Sanhueza J, De la Barra F. El aceite de pescado: ayer un desecho industrial, hoy un producto de alto valor nutricional. Rev Chil Nutr. 2012; 39: 201-9.

13. Henderson R, Burkow I, Buzzi M, et al. Effects of docosahexaenoic, tetracosapentaenoic and tetracosahexaenoic acids on the desaturation and elongation of $n-3$ polyunsaturated fatty acids in trout and salmon liver microsomes. Biochim Biophys Acta. 1998; 1392: 309-19.

14. Valenzuela A. El Salmón: un banquete de salud. Rev Chil Nutr. 2005; 32: 8-17.

15. Valenzuela $A$, Yáñez C, Golusda C. El ostión del norte chileno (Argopecten purpuratus), un alimento de alto valor nutricional. Rev Chil Nutr. 2011; 38: 148-55.

16. Valenzuela A. Aceites de origen marino y su importancia en la salud y nutrición humana y animal. Aceites Grasas. 2009; XIX: 154-61.

17. Swanson D, Block $R$, Mousa S. Oemag-3 fatty acids EPA and DHA: Health benefits throughout life. Adv Nutr. 2012; 3: 1-7.

18. Sorongon-Legaspi $M$, Chua $M$, Sio M, Morales $M$. Blood level omega-3 fatty acids as risk determinant molecular marker for prostate cancer. Prostate Cancer. 2013; 4: 13-22.

19. Chua $M$, Sio $C$, Sorongon $M$, et al. The relevance of serum levels of long chain omega-3 polyunsaturated fatty acids and prostate cancer risk: A meta-analysis. Can Urol Assoc J. 2013; 7: e333-e43.

20. Taneja A, Singh H. Challenges for the delivery of long-chain fatty acids in functional foods. Ann Rev Food Sci Technol. 2012; 3: 105-23.

21. Drusch S, Mannino S. Patent based review on industrial approaches for the microencapsulation of oils rich in polyunsaturated fatty acids. Trends Food Sci Technol. 2009; 20: 237-44.

22. Dekker $R$, Husken $H$. Encapsulated omega-3 fatty acids 
and/or omega- 6 fatty acids and /or esters thereof with a coating. U.S. Patent N 2010/0285118 A1.

23. Valenzuela A, Sanhueza J, Nieto S. Docosahexaenoic acid $(D H A)$, essentiality and requirements: Why and how to provide supplementation. Grasas Aceites. 2006; 57: 229-237.

24. Lawson L, Hughes B. Human absorption of fish oil fatty acids as triacylglycerols, free fatty acids, or ethyl esters. Biochim Biophys Acta. 1988; 152: 328-35.

25. Chen B, McClements D, Decker E. Design foods with bioactive lipids for improved health. Annu Rev Food Sci Technol. 2013; 4: 35-56.

26. Adarme-Vega C, Lim D, Timmins M, et al. Microalgal biofactories: a promising approach towards sustainable omega-3 fatty acid production. Microbial Cell Fact. 2012: 11: 96-105.

27. Berenhauser A, Pinheiro do Campo A, Claro da Silva $R$, et al. Fattya cid composition in preterm and term breast milk. Int J Food Sci Nutr. 2012; 63: 318-25.

28. Parmentier M, Al Sayed M, Linder M, Fanni J. Polar lipids: $n-3$ PUFA carriers for membranes and brain: nutritional interest and emerging processes. OCL 2007;14: 224- 229.

29. Tou J, Jaczynski J, Chen Y. Krill for human consumption: nutritional value and potential health benefits. Nutr Rev. 2007; 65: 63-77.

30. Choi H, Kim J, Chang $M$, et al .Effects of astaxantin on oxidative stress in overweight and obese adults. Phytother Res. 2011; 25: 1813-18.

31. Burri L, Hoem N, Banni S, et al. Marina omega-3 phospholipids: metabolism and biological activities. Int $\mathrm{J} \mathrm{Mol}$ Sci. 2012; 13: 15401-19.

32. Barceló-Coblijn G, Murphy E. Alpha-linolenic acid and its conversion to longer chain n-3 fatty acids: Benefits for human health and a role in maintaining tissue $n-3$ fatty acids levels. Prog Lipid Res. 2009; 48; 355-74.

33. Morales J, Valenzuela R, González D, González M, Tapia $G$, Sanhueza J, Valenzuela A. Nuevas fuentes dietarias de ácido alfa linolénico: una visión crítica. Rev Chil Nutr. 2012; 39: 79-87.

34. Valenzuela $R$, Gormáz J, Masson L, Vizcarra M, Cornejo P, Valenzuela A, Tapia G. Evaluation of hepatic bioconversion of alpha linolenic acid ( $A L N$ ) to eicosapentaenoic acid (EPA) and docosahexaenoic acid (DHA) in rats fed oils from chia (Salvia hispanica) and Rosa mosqueta (Rosa rubiginosa). Grasas Aceites. 2012; 63: 61-69.

35. Gonzalez D, Tapia G, Gormaz J, Despessailles A, Espinoza $A$, Masson $L$, Varela $P$, Valenzuela $A$, Valenzuela $R$. Bioconversion of alpha-linolenic acid to $n-3$ LCPUFA and expression of PPAR-alpha, acyl coenzyme A oxidase 1 and carnitine acyl transferase I are incremented after feeding rats with alpha-linolenic acid-rich oils. Food Function. 2012; XX: 1-9. 